\title{
The dilemma and the improvement path of the proposition situation of science in Chinese college entrance examination
}

\author{
Wenhui Gong ${ }^{1 *}$, Hongjun Xing ${ }^{2}$ \\ ${ }^{1}$ College of Education, Capital Normal University, China, 100048 \\ ${ }^{2}$ College of Teacher Education, Capital Normal University, China, 100048 \\ *Correspondence: 18335182783@163.com
}

(Received: 09/30/2019; Accepted: 11/17/2019; Published: 11/20/2019)

DOI: https://doi.org/10.37906/real.2019.3

\begin{abstract}
As the reform of the examination enrollment system and the examination content in China evolve, although we have witnessed some progress in the quality of science college entrance examination proposition, some challenges and problems persist, mainly in: inadequate diversity of situation, insufficient presentation of the situation and contempt for the scientific nature of situation. In view of the above issues in the compilation of proposition situation, the following improvements are proposed: select situation scientifically, present situation effectively, and explore whether the situation follows the nature of science.
\end{abstract}

Keywords: proposition situation of science; science education; college entrance examination

\section{Introduction}

Traditional knowledge view assumes that learning is a process that can be separated from the situations in which it is used, treating knowledge as a singular and self-sufficient entity, which can be transferred from instructors to learners. In the traditional school education, the learner is isolated from the real environment, "learning" and "doing" are separated. Schools focus on abstract, simplified and decontextualized concepts (Brown, Collins, \& Duguid, 1989). These concepts promote understanding that is rigid, incomplete, and naive (Spiro, Feltovich, Jacobson, \& Coulson, 1991). By the late 1980s, some researchers began to challenge the assumption that learners were separated from situation. According to Newmann, "People in diverse fields face the primary challenge of producing, rather than reproducing, knowledge. This knowledge is expressed through discourse, through the creation of things, and through performance." (Newmann, 1991) In order to develop the skills used by experts for students, authentic tasks in authentic contexts should be provided for them to engage in (Choi \& Hannafin, 1995). Brown et al. (Brown, Collins, \& Duguid, 1989) believe that knowledge is subject to the basic influence of the activities, situations and cultures in which knowledge is used, and cannot be separated from them.

With the continuous development of the situated cognition theory, the importance of the situation is emphasized increasingly in many aspects of education, such as instructional design and educational evaluation. In particular, the significance of the situation in the examination was emphasized by the government document Guiding opinions on promoting the reform of education mode of ordinary high schools in the new era issued by General Office of the State Council in 2019 (General Office of the State Council, 2019). The document proposed that innovative test forms should be promoted, design of test situation should be 
strengthened, and comprehensive, open, applicable and exploratory test questions should be included. The general senior secondary school chemistry curriculum standards (the 2017 version) released by the Ministry of Education (MoE) in January 2018 (Ministry of Education, 2018), as one of the main bases of the examination proposition, assigns great importance to the proposition situation, and puts forward a specific proposition framework, which was depicted in Figure 1.

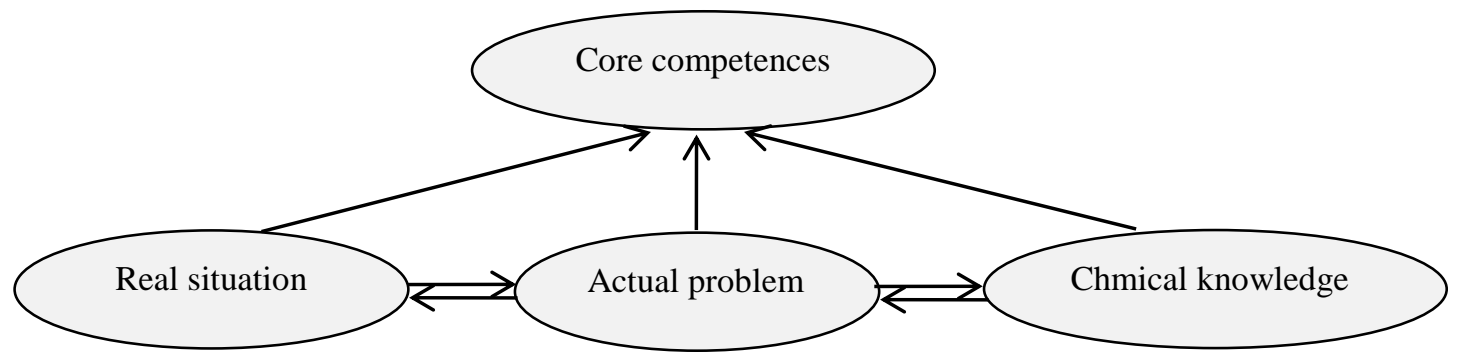

Figure 1. Framework of proposition

The framework was constructed based on the core competencies in the subject of chemistry, which were called the "chemistry core competencies", including macroscopic identification and microscopic analysis, change conception and equilibrium thought, evidence-based reasoning and modeling cognition, scientific inquiry and innovation consciousness, scientific attitude and social responsibility. Four principles were manifested in the framework, namely, (1) Taking core literacy as testing purpose, (2) Taking the real situation as the test carrier, (3) Taking practical problems as test tasks, (4) Taking chemical knowledge as problem-solving tools. The above principles aimed at fulfilling the fundamental tasks of "lide shuren", which was promulgated by MoE in 2014 (Ministry of Education, 2014). To achieve the goal, the proposition situation, chemical knowledge and problem-solving should serve the examination of students' chemical core literacy. Moreover, students' ability to solve "practical problems" depends on their capacity to apply "chemical knowledge" in "real situation".

Despite the continuous evolution of the reform of the examination enrollment system and the reform of the examination content, there are various types of the examination situation and the gradual improvement of the quality of the proposition, but some imperfections still exist. In view of the dilemma encountered in the proposition situation, this paper tries to put forward some paths for improvement.

\section{Dilemma of the proposition situation of science in Chinese college entrance examination}

Through literature review and analysis of some test questions of science in Chinese college entrance examination, some problems have been found as follows: inadequate diversity of situation, insufficient presentation of the situation, contempt for the scientific nature of situation.

\subsection{Inadequate diversity of situation}

At present, strengthening the design of the proposition situation has not only reached a consensus in the academic class, but also has risen to the policy level. Generally, researchers believe that "situation" is characterized by authenticity, practicality and sociality. However, the understanding of "authenticity" is still controversial. For example, some researchers believe that the authenticity of the situation is refers to the person on which exist in the real-world situation, rather than imaginary or fictional situation. Accordingly, they hold the idea that the proposition of test should be based on real material, combined 
with living example, examines students' basic knowledge and problem-solving ability, and to help students transfer the knowledge from one situation to another (Yu \& Zhang, 2019). Apparently, this kind of view emphasizes the "reproduction" of the real situation. Different from this opinion, some researchers suggested to further expand the meaning of "real", confirmed that the situation can be fictional, but the situation should have authenticity (Zhang \& Hu, 2016), which means that the situation designed must be reasonable, i.e. it can be achieved.

The theory of situational cognition can give suggestions for us, which claimed that learning is the process of creating meaning in the interaction between individuals and the situation. Learning should be carried out in specific and meaningful situations, and it will be profoundly influenced by specific task or problem situations (Brown, Collins, \& Duguid, 1989). When selecting the real situation for evaluation, we should not only focus on the similarity or fidelity between the current situation and the target situation in real life (Herrington \& Herrington, 1998). This is because that realistic tasks and the conditions under which they are carried out are difficult to reproduce completely. Even if the researcher is very careful, there are many variables or details will be lack of control (Messick, 1994). Complicating matters is the fact that authenticity is subjective and is dependent on perceptions (Gulikers, Bastiaens, \& Kirschner , 2004), which indicates that students, teachers, and evaluators may differ in their understanding of the concept of authenticity. Although teachers and evaluators may do their best to develop authentic assessments, this may all be for nothing if the student does not perceive them as such. This process is known as "preauthentication" (Huang, 2002; Petraglia, 1998). The fact that certain situations cannot be replicated may be the biggest problem faced by the "authenticity" feature of the situation. Situation, therefore, don't have to be limited to replicating life scenes that have already happened. The test designers can modify the test context appropriately according to the test purpose. They can fully carry out "thought experiment", conceived a virtual-reality occur at a certain time and space in their minds, and to ensure that this situation must be "reasonable", which means that the situation accords with scientific knowledge and scientific methods, and has positive scientific value and educational significance. Hence, the "authenticity" of the situation should keep moving towards "rationality", so as to avoid the "narrowing" of the situation and reveal the true meaning of "situation". Only in this way can the diversity of the situation be enriched.

Innovation is an important factor to judge the situation quality of test questions. However, innovative situations are relative to different students, which is mainly reflected in that the situation is "new" to some students, but not "novelty" to others. This phenomenon shows the urbanized tendency of the context of proposition, which means that, some abilities that the questions aim to test are hard to obtain completely depending on the individual efforts of students, but depend heavily on the quality of educational conditions. In order to avoid the urbanization risk of proposition and realize the fairness of proposition, it is a great challenge for proposition makers to choose and adapt the content and situation of the test questions.

\subsection{Insufficient presentation of the situation}

Due to the different backgrounds among teachers, educators and students, different people have various interpretations of even the same test situation. Some questions of science curriculum in the college entrance examination of China have abundant meanings, but they are not clearly stated, so that students can hardly understand the significance of the situation and the educational function of test situation cannot be fully manifested.

Taking the question 28. (3) of science comprehensive college entrance examination (national I roll) in 2019 as an example. The situation of the question is "Chinese scholars have studied the reaction process of water-gas transformation on the surface of gold catalyst by combining experimental and computer 
simulation results." In fact, the situation is adapted from an article published by Chinese scholars in the top international journal of Science, the title of the article is "Atomic-layered Au clusters on $\alpha-\mathrm{MoC}$ as catalysts for the low-temperature water-gas shift reaction" (Yao, et al., 2017). The purpose of choosing the theoretical calculation model of single atom catalytic energy change as the situation is to enable students to understand and analyze the catalytic adsorption mechanism and energy change in the reaction process, and to feel the application of modern information computer simulation technology in chemical research (National Education Examinations Authority, 2019). Obviously, the context of the test is limited to a simple objective description of the scientific method and scientific research process, the significance of this important scientific research achievement is not highlighted, and the potential sufficient significance of this great discovery has been obscured. If the significance of this discovery could be clearly stated in the context: "The traditional water-gas conversion reaction has high temperature and high energy consumption. Chinese scientists have proposed an efficient catalytic technology, namely the single atom catalytic method, which enables the water-gas conversion reaction to be carried out at a temperature of $303 \mathrm{~K}$, far lower than that in other existing catalytic methods." Only in this way can the scientific significance of the situation compiled by the propositional personnel be fully demonstrated, so that students can experience the progress of science and technology in China, better understand the great achievements made in China, and enhance their national pride.

Sometimes, in order to highlight the sufficient meaning of the situation, it may go to the other extreme of "excessive information", which will not only waste students' time in reading and reviewing the questions, but also increase their memory burden, which violates the original intention of the proposition and also reduces the validity of the test questions. What's worse, the information contained in the situation does not directly provide useful information for students to solve the problem. In this case, the situation will seem a little excessive, and may lead to a lot of negative test-taking skills, such as skipping the situation, looking directly at the problem, and then solving the problem. To prevent these extreme conditions from happening, key problem-solving information should be integrated into the propositional situation, which can not only achieve the educational function of the situation, but also can truly test the students' problem-solving ability.

The other performance of insufficient presentation of the situation is the deficiency openness of situation. Taking the question 31 (see figure 2) of science comprehensive college entrance examination (national II roll) in 2019 as an example. The question is designed to address the energy problem of the ecosystem, and is positioned to "examines the integrated understanding of photosynthesis and respiration in plants, and measures the students' ability to analyze and solve problems comprehensively, reflecting the comprehensive requirements" (National Education Examinations Authority, 2019) by the National Education Examinations Authority. 
Answer the following questions about ecosystems.

(1)In forest ecosystems, the energy of producers comes from and can flow directly to (You only need to answer 2 key points).

(2)In general, for an aquatic ecosystem, the total primary production of phytoplankton (the amount of organic matter produced by producers) can be calculated based on changes in oxygen content in the water. To determine the total primary production of phytoplankton in an aquatic ecosystem, water samples can be taken from the depths of a certain water in the aquatic ecosystem and divided into three equal parts. The other two bottles are put into two glass bottles which are impermeable to light (a) and transparent to light (B) respectively, sealed and put back to the sampling place. After several hours, the content of $\mathrm{O}_{2}$ in bottle a (B) and the content of $\mathrm{O}_{2}$ in bottle $\mathrm{B}$ (C) are determined. Answer the following questions accordingly.

Under the condition that producer respiration is the same in bottles $a$ and $b$ and only the producer is in bottles, In this experiment, the difference between $C$ and $A$ indicates that ; the difference between $C$ and $B$ indicates that ; the difference between $\mathrm{A}$ and $\mathrm{B}$ indicates that

Figure 2. Question 31 of science comprehensive college entrance examination (national II roll) in 2019

Obviously, the first question is about knowledge. The degree of the situation structure of second item is relatively high, not only provides calculating total primary production of phytoplankton in ecological system, the method of the oxygen content of the changes, and also gives the specific operation steps. Finally, students were left with very little freedom to answer the questions. Students were asked to give a fixed answer: "the amount of oxygen released by the producer for net photosynthesis". "Total oxygen emission from producer photosynthesis"; Oxygen consumption for producer respiration. Moreover, a series of "thinking crutch" provided by the test questions weaken the aim of measuring students' ability to analyze and solve problems comprehensively. Since most of the thinking process of students' analysis and solving problems has been completed by the test designers, the requirement of "comprehensiveness" cannot be fully measured in the end. As a result, only by coordinating the relationship among test context, the degree of question structure, and the level of students' freedom to answer questions, can the purpose of the proposition be fully manifested, and the key competencies be fulfilled.

\subsection{Contempt for the scientific nature of situation}

In the current college entrance examination questions, whether the situation follows the nature of science has not been paid enough attention. On the one hand, the situation itself is against the scientific fact. Taking the question 13 of science comprehensive college entrance examination (Guang Dong roll) in 2013 (see figure 3) as an example. 
An aircraft carrier runway length is 200 meters. The maximum acceleration of the aircraft taxiing on the aircraft carrier is $6 \mathrm{~m} / \mathrm{s}^{2}$, and the minimum speed required for take-off is $50 \mathrm{~m} / \mathrm{s}$. Then, the minimum speed that the aircraft needs to obtain with the help of the ejection system before taxiing is ( )
(A) $5 \mathrm{~m} / \mathrm{s}$
(B) $10 \mathrm{~m} / \mathrm{s}$
(C) $15 \mathrm{~m} / \mathrm{s}$
(D) $20 \mathrm{~m} / \mathrm{s}$

Figure 3. Question 13 of science comprehensive college entrance examination (Guang Dong roll) in 2019

By substituting the given data in the test context into the relevant physical formula, it can be calculated that the answer to the question is B. The calculation process of this problem is very scientific and rigorous, in line with the theoretical requirements. But the context of the problem itself contradicts scientific fact. For the reason that, in general, the minimum speed required for fighter jets on an aircraft carrier to take off is about $70 \mathrm{~m} / \mathrm{s}$, while the speed of the aircraft carrier itself is about $15 \mathrm{~m} / \mathrm{s}$ in the process of operation. Therefore, the minimum speed of fighter jets when taking off relative to the aircraft carrier is about $50 \mathrm{~m} / \mathrm{s}$, and its absolute speed is about $70 \mathrm{~m} / \mathrm{s}$. Unless otherwise specified, people usually use the ground as a reference frame when describing the motion of an object. In this question, "the minimum speed required for take-off is $50 \mathrm{~m} / \mathrm{s}$ ", which is understood from the perspective of the default reference frame selection rule or the specific context of the question, is based on the ground as the reference frame (Song \& Hu, 2019).

On the other hand, the situation itself has no problem, but the deeper science nature behind it is ignored. Taking the question 7 (see figure 4) of science comprehensive college entrance examination (national I roll) in 2019 as an example.

Ceramic is the crystallization of fire and soil, and is one of the symbols of Chinese civilization. Its formation and nature are closely related to chemistry. The following statement is incorrect ( )

A. "Rain stopped, dark cloud faded to welcome a cyan sky" describes the cyan color of porcelain, which comes from iron oxide

B. The world-famous terra-cotta warriors of the qin dynasty are pottery products made of clay sintered at high temperature

C. Ceramics are early artificial materials, the main chemical composition is silicate

D. The ceramic has stable chemical properties, acid and alkali corrosion resistance, oxidation resistance and other advantages

Figure 4. Question 7 of science comprehensive college entrance examination (national I roll) in 2019

Students generally learned that the color of iron oxide is red, so they will infer that the cyan is not from iron oxide, and the item of A is incorrect. The fact is, however, that the composition of porcelain is very complicated, such as cobalt oxide, manganese oxide and iron oxide etc., and the origin of the cyan of porcelain is also very diverse. Some porcelain, such as the imperial porcelain of song dynasty, is added 
with blue glaze, which mainly contains iron, titanium and manganese, the content of $\mathrm{Fe}_{2} \mathrm{O}_{3}$ is relatively high, while that of $\mathrm{TiO}_{2}$ and $\mathrm{MnO}$ is relatively low. Moreover, titanium and manganese have limited influence on the color of porcelain, it is mainly colored with iron. With the increase of $\mathrm{Fe}_{2} \mathrm{O}_{3}$ content, the glaze color changes from azure and pink to grey (Ding, Zhao, Huang, Hou , \& Miao, 2010). That is to say, although the porcelain of different ages and types is cyan in the end, there are slight differences in the quality, tone, gradation and interpretation of the porcelain, which also reflects the aesthetic art and unique craftsmanship of the ancient Chinese working people. Different materials in the porcelain are mixed according to different proportions, and under certain conditions will present a variety of cyan tones, and iron oxide is indeed one of them. Therefore, the cyan color of porcelain does have a contribution from iron oxide, so the option A should be correct. As a result, there is no correct answer to this question, which means that this question is not rigorous and scientific. The reason that leads to this result is the test designers' negligence of the scientific nature contained in the situation itself. Superficially, the question highlights the subject value of chemistry, taking Chinese traditional culture and traditional technology as the context of inheriting Chinese scientific culture. However, the creation of such an "innovative" situation is at the expense of being "scientific", which is against the nature of science. Obviously, this kind of question does not accord with the original intention of the proposition.

\section{Improvement paths and its implications}

In view of the above problems existing in the situation design of the current proposition, the following improvement paths are proposed.

\subsection{Select situation scientifically}

One of the important reasons why alternative and novel situations are not enough is that the situation types of propositions are too limited to "authenticity". Therefore, the types of propositional situations should be constantly enriched. When the situation changes from "real" in the narrow sense to "reasonable" in the broad sense, the selection of the situation has obtained infinite possibilities. Taking Context-Rich Problems as an example (Heller \& Hollabaugh, 1992; Enghag, Gustafsson, \& Jonsson, 2009):

- Each problem is a short story that include a reason (if sometimes far-fetched and humorous) for calculating something.

- The major character in each problem is the student.

- The objects in the problems are real or can be imagined.

- Students need to actively visualize the situation using their own experience.

- The problem cannot be solved in one step by plugging numbers into a formula.

Certainly, the situation types of Context-Rich Problems are very rich for the reason that the situations are not only limited to the real, but also emphasize the imaginable. It must be emphasized that this kind of imaginability is not a myth without scientific basis, but a reasonable problem that can be solved successfully by means of hypothesis, scientific reasoning, demonstration, negotiation, etc. on the basis of scientific principles.

When compiling the propositions, great importance should also be attached to the unique function of traditional culture to build up morality and cultivate people, all the subjects are required to undertake the mission of carrying forward and examining China's excellent traditional culture to reflect the great mission of selecting talents for the country. When it comes to the process of designing the test questions of science curriculum, relatively representative ancient Chinese science and technology civilization can be selected to reflect the contribution of traditional Chinese science and technology culture to human 
development and social progress (Jiang, Editorial Department of China Examinations, \& National Education Examinations Authority, 2017). Such as question 7 of science comprehensive college entrance examination (national I roll) in 2019, question 12 of science comprehensive college entrance examination (national II roll) in 2019, question 7 of the physics part of science comprehensive college entrance examination (Tian Jin roll) in 2018, taking the materials that can represent Chinese traditional science and technology civilization, such as ceramic, inorganic pigments and the story of "Wedge the tower body" recorded in "five miscellaneous group" written by Zhaozhe Xie in the Ming dynasty as context materials respectively to examine the scientific knowledge embodied therein.

It should be noted that it is necessary to examine the scientific knowledge reflected in the achievements of China's ancient scientific and technological civilization. However, it is difficult to reflect the development and innovation of traditional culture in reality by merely recalling China's excellent traditional culture. Therefore, to test China's excellent traditional culture in the proposition, it is key to follow the development path of inheritance, promotion and innovation, carry out innovation at the same time, pay attention to the creative transformation and innovative development of traditional culture in reality, so as to realize the social significance and realistic purpose of the exam.

\subsection{Present situation effectively}

In order to avoid the two extreme phenomena of redundant or insufficient information provided by the proposition situation, it is necessary to ensure the appropriate load of situation information, although this problem needs to be analyzed according to the actual situation. In addition, it is very necessary to control the degree of freedom of students to solve problems by adjusting structural extent of the situation, so as to provide students with appropriate space for reasoning and argumentation.

While it is generally accepted that most real-life problems are open and complex, and that it is necessary for students to learn to solve such problems, there are almost no such propositions in china. The context and questions are highly structured with insufficient openness. In fact, there is a gradually changing continuum between completely closed multiple choice questions and completely open tasks (Messick, 1994). The key dimension of this change is the degree of structure of the situation and the degree of freedom for students to complete the task (see figure 5) (Yang, 2015). 


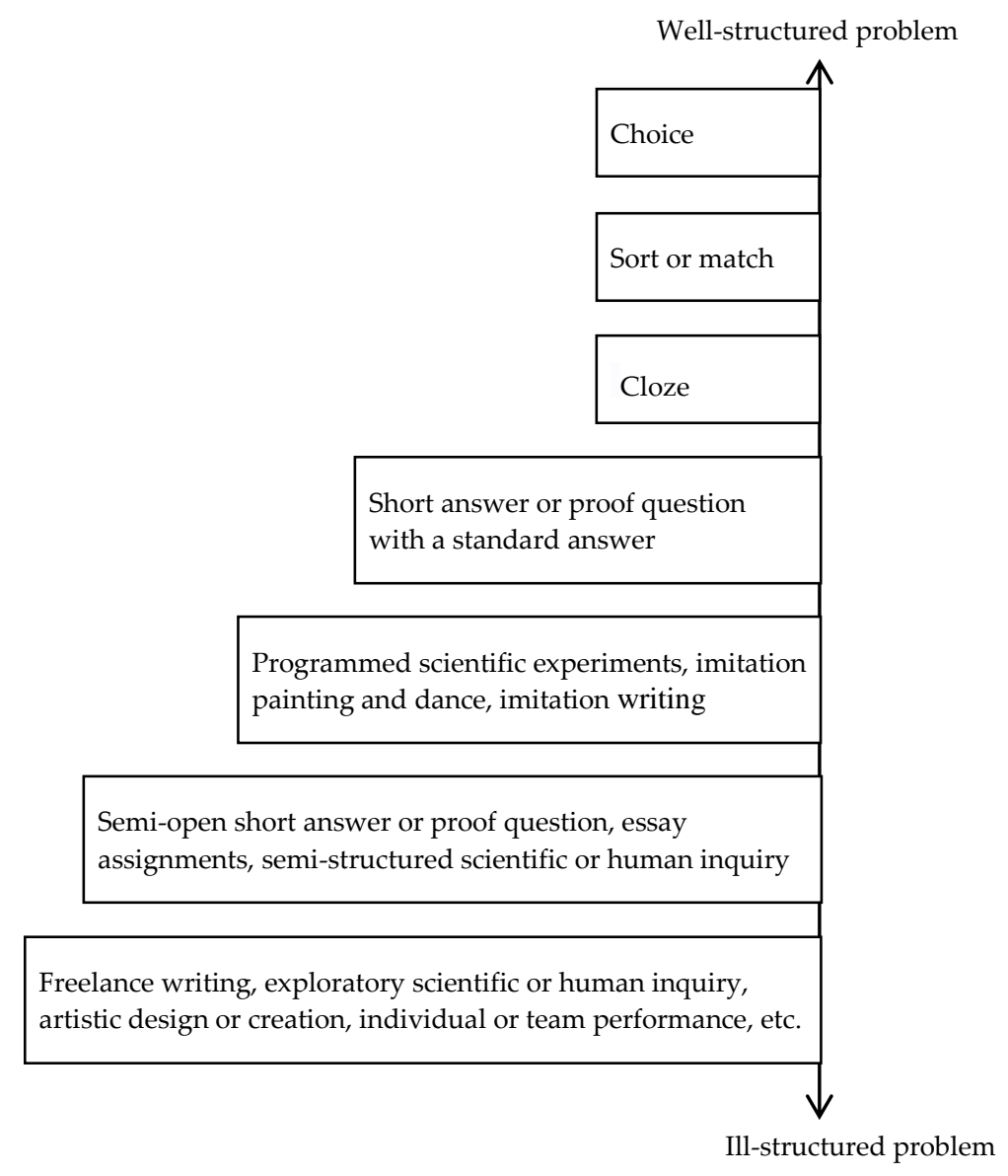

Figure 5. Continuum of well-structured and ill-structured problem

Certainly, the open degree of situation is inversely proportional to the degree of freedom for students to complete the question. Thereafter, the relationship between the situation need to be designed reasonably in accordance with the objective of proposition to improve the effectiveness of test, and verify that the problems follow the nature of science.

\subsection{Explore whether the situation follows the nature of science}

In the past years, people have paid more attention to whether the problem follows the nature of science. Although the significance of the situation is more and more recognized by people of insight, however, the situation is often regarded as only the background of the problem, and its form is considered more important than its content. Therefore, in the pursuit of novelty of the situation, the scientific nature of the test will be ignored sometimes. This leads to a typical phenomenon, that is, situation and problem are separated or weakly related. This situation reflects the researchers' insufficient attention to the situation and the lack of in-depth exploration of the scientific nature of the situation itself. In this sense, both innovation and whether the situation follows the nature of science need to be considered, and the situation should be corrected and perfected in time.

The selected situation is too complicated and difficult for students sometimes, which may lead to the alienation of the goal of the test. For example, the composition of ceramic is so complex that scientists 
have not yet been able to understand the chemical substances and other relevant scientific principles contained in it, and the proposition personnel lacked a deeper analysis of the situation, but just simply related the situation to the problem, that is, the iron oxide was associated with the color of ceramics, making the mistake of equating "porcelain cyan" with "single cyan" and ignoring the scientific fact that the composition of porcelain cyan was diverse. Due to the reason that the successful solution of the corresponding question is directly related to the composition of ceramics, this question is contrary to scientific nature.

In conclusion, whether the proposed situation follows the nature of science should be explored deeply so as to make it consistent with the purpose of the proposition. Scientific selection and compilation of innovative situation will become important directions to improve the level of proposition in the near future.

Funding: "This research was funded by the General Project of Education of the National Social Science Foundation of China: "Research on the Composition and Performance of Key Competence, grant number BBA190024"

Acknowledgments: The authors gratefully acknowledge the editor of REAL for his time and invaluable suggestions, which elevate the quality of this paper.

Conflicts of Interest: The authors declare no conflict of interest.

\section{References}

Brown, J. S., Collins, A., \& Duguid, P. (1989). Situated cognition and the culture of learning. Educational Researcher. 18(1), 32-42.

Choi, J. I., \& Hannafin, M. (1995). Situated cognition and learning environments: roles, structures, and implications for design. Educational Technology Research and Development, 43(2), 53-69.

Ding, Y., Zhao, L., Huang, W., Hou , J., \& Miao, J. (2010). Nondestructive Determination of the Glaze Colors of the Song-dynasty Guan Wares Collected in the Palace Museum (In Chinese). Palace Museum Journal, 65(05), 146-152.

Enghag, M., Gustafsson, P., \& Jonsson, G. (2009). Talking physics during small-group work with contextrich problems-analysed from an ownership perspective. International Journal of Science and Mathematics Education, 7(3), 455-472.

General Office of the State Council. (2019). Guiding opinions on promoting the reform of education mode of ordinary high schools in the new era (In Chinese). Retrieved from http://www.gov.cn/xinwen/201906/19/content_5401610.htm

Gulikers, J. T., Bastiaens, T. J., \& Kirschner , P. A. (2004). A five-dimensional framework for authentic assessment. Educational Technology Research and Development, 52(3), 67-86.

Heller, P., \& Hollabaugh, M. (1992). Teaching problem solving through cooperative grouping. Part 2: Designing problems and structuring groups. American journal of Physics, 60(7), 637-644. 
Herrington, J., \& Herrington, A. (1998). Authentic assessment and multimedia: how university students respond to a model of authentic assessment. Higher Education Research \& Development, 17(3), 305322.

Huang, H. (2002). Toward constructivism for adult learners in online learning environments. British Journal of Educational Technology, 33(1), 27-37.

Jiang, G., Editorial Department of China Examinations, \& National Education Examinations Authority. (2017). Steadily Promoting the Content Reform of the College Entrance Examination to Help Improve the Quality of Education: An Interview with JIANG Gang,Director of the National Education[J] (In Chinese). China Examinations, 15(01), 1-7.

Messick, S. (1994). The interplay of evidence and consequences in the validation of performance assessments. Educational Researcher, 23(2), 13-23.

Ministry of Education. (2014). Opinions on deepening curriculum reform and implementing the fundamental tasks of lide shuren (In Chinese). Retrieved from http://old.moe.gov.cn/publicfiles/business/htmlfiles/moe/s7054/201404/167226.html

Ministry of Education. (2014). The Ministry of Education's opinions on the implementation of the general high school academic level examination (In Chinese). Retrieved from http://old.moe.gov.cn//publicfiles/business/htmlfiles/moe/s4559/201412/181664.html

Ministry of Education. (2018). The general senior secondary school chemistry curriculum standards (the 2017 version) (In Chinese). People's Education Press: Beijing.

National Education Examinations Authority. (2019). Illustrate Biological Characteristics and Commit Cultivation Function:Analysis of the Biology Test of 2019 Gaokao (In Chinese). China Examinations, 17(07), 25-28.

National Education Examinations Authority. (2019). Select Item Context and Deepen Gaokao Content Reform:Analysis of the Chemistry Test of 2019 Gaokao (In Chinese). China Examinations, 17(07), $20-24$.

Newmann, F. M. (1991). Linking restructuring to authentic student achievement. The Phi Delta Kappan, 72(6), 458-463.

Petraglia, J. (1998). Reality by design: The rhetoric and technology of authenticity in education. Mahwah. NJ: Lawrence Erlbaum Associates Publishers.

Song, Y., \& Hu, Y. (2019). A case study on the science of the situation of physics curriculumof college entrance examination (In Chinese). Physics Teacher, 40(06), 71-73.

Spiro, R. J., Feltovich, P. J., Jacobson, M. J., \& Coulson, R. S. (1991). Knowledge representation, content specification, and the development of skill in situation-specific knowledge assembly: Some constructivist issues as they relate to cognitive flexibility theory and hypertext. Educational Technology, 31(9), 22-25.

State Council. (2014). The implementation opinions on deepening the reform of the examination and enrollment system (In Chinese). Retrieved from http://www.gov.cn/zhengce/content/201409/04/content_9065.htm

Yang, X. (2015). Toward a Construct-driven Approach to Authentic Assessment (In Chinese). Global Education, 44(05), 36-49. 
Yao, S., Zhang, X., Zhou, W., Gao, R., Xu, W., Ye, Y., . . Ma, D. (2017). Atomic-layered Au clusters on $\alpha-$ $\mathrm{MoC}$ as catalysts for the low-temperature water-gas shift reaction. Science, 357(6349), 389-393.

Yu, X., \& Zhang, Y. (2019). Compilation and improvement of middle school physics examination questions from the perspective of scientific literacy -- a case study of physics academic level examination in shandong province in 2018 (In Chinese). Education measurement and evaluation, 12(05), 39-44.

Zhang, Q., \& Hu, B. (2016). Situational knowledge and contextualized curriculum design (In Chinese). Curriculum,Teaching Material and Method, 36(06), 26-32. 